\title{
Overexpression of TROP2 promotes proliferation and invasion of ovarian cancer cells
}

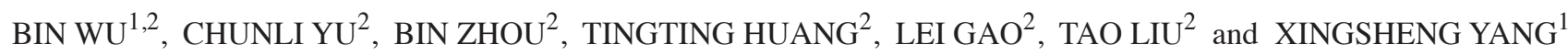 \\ ${ }^{1}$ Department of Gynecology, Qilu Hospital, Shandong University, Jinan, Shandong 250012; \\ ${ }^{2}$ Department of Gynecology, Taian City Central Hospital, Taian, Shandong 271000, P.R. China
}

Received April 18, 2016; Accepted March 31, 2017

DOI: $10.3892 /$ etm.2017.4788

\begin{abstract}
Human trophoblastic cell-surface marker, tumor-associated calcium signal transducer 2 (TROP2), is a newly identified marker that has a vital role in the proliferation and invasion of various tumors. However, its specific function in ovarian cancer has not been researched. The purpose of the present study was to investigate the role of TROP2 in the formation of ovarian cancer and its possible mechanism. TROP2 was knocked down by small interfering (si)RNA in ovarian cancer cell line, A2780. The expression of TROP2 protein following transfection was detected by western blot analysis. Cell viability was determined using a Cell Counting kit-8. Cancer cell migration and invasion were examined by wound healing and cell invasion assays, respectively. Apoptosis-related proteins, such as B-cell lymphoma 2 (Bcl-2) and Bcl-2-associated X protein (Bax), were measured by western blotting. Results demonstrated that the expression levels of TROP2 were markedly downregulated by siRNA in A2780 cells compared with the control groups, which led to strong inhibition of proliferation and invasion. Furthermore, TROP2 downregulation also reduced cell migratory ability. Additionally, in the TROP2-knockout group, Bcl-2 was downregulated and Bax was upregulated compared with the control. The present study suggested that the expression of TROP2 was related to cellular proliferation, migration and invasion. TROP2 may disrupt the balance in the Bax family to participate in apoptosis regulation in A2780 cells. Therefore, the overexpression of TROP 2 may have a crucial role in tumorigenesis and tumor progression by disturbing the $\mathrm{Bax} / \mathrm{Bcl}-2$ balance in ovarian cancer.
\end{abstract}

Correspondence to: $\mathrm{Dr}$ Xingsheng Yang, Department of Gynecology, Qilu Hospital, Shandong University, 107 Wenhuaxilu Road, Jinan, Shandong 250012, P.R. China

E-mail: xingshengyang@sdu.edu.cn

Key words: tumor-associated calcium signal transducer 2, ovarian cancer, proliferation, migration, small interfering RNA

\section{Introduction}

Ovarian cancer is one of the most common malignancies in women. Though its morbidity ranks third after cervical cancer and endometrial carcinoma among female genital system neoplasms, the mortality of ovarian cancer tops the list (1). In 2014, ovarian cancer was responsible for $\sim 21,980$ new cases and $\sim 14,270$ deaths in the United States (1). Owing to the complexity of the ovary in embryogenesis, the anatomical structure and reproductive endocrine function, early stage ovarian cancer is often hard to detect in patients (2). The majority of ovarian cancer cases are epithelial ovarian carcinomas, which are often diagnosed at the advanced stage, leading to very poor prognosis (3). Tumor-associated calcium signal transducer 2 (TROP2), which is differentially expressed in various types of cancer, is a transmembrane glycoprotein encoded by the TACSTD2 gene (4). TROP2 was initially discovered in trophoblast cells, specifically in chorionic trophoblast cells during placental implantation, and may promote the invasion and metastasis of tumor cells $(5,6)$. Overexpression of TROP2 has been identified in breast cancer (7), colorectal cancer (8), endometrial endometrioid adenocarcinoma (9), cervical cancer (10), laryngeal carcinoma (11) and oral squamous cell cancer (4). Therefore, TROP2 may be a potential prognosis biomarker, as well as a novel therapeutic target. Although research exits on TROP2 expression and poor survival in Italian patients with ovarian carcinoma (12), the biological significance of TROP2 in epithelial ovarian carcinomas remains unclear. It has previously been demonstrated that TROP2 protein was overexpressed in ovarian cancer cases and its expression level was correlated with the clinical outcomes in another previous study $(13,14)$. The present study aimed to investigate the specific function of TROP2 in ovarian cancer cells. The effects of TROP2 expression on proliferation, cell invasion and metastasis were investigated in vitro.

\section{Materials and methods}

Cell culture. A2780, HO8910 and SK-OV-3 cell lines were purchased from the American Type Culture Collection (Manassas, VA, USA). Cells were cultured at $37^{\circ} \mathrm{C}$ in RPMI 1640 medium (Hyclone, GE Healthcare Life Sciences, Logan, 
UT, USA) supplemented with $10 \%$ mycoplasma-free fetal bovine serum (FBS; Gibco; Thermo Fisher Scientific, Inc., Waltham, MA USA) and $1 \%$ penicillin-streptomycin in a $\mathrm{CO}_{2}$ incubator under standardized conditions.

Immunofluorescence staining. The three cell lines were seeded at 35,000 cells per well onto $15-\mathrm{mm}$ glass slides in a 24 -well plate and incubated at $37^{\circ} \mathrm{C}$ for $24 \mathrm{~h}$. Cells were fixed at room temperature with $4 \%$ paraformaldehyde for $15 \mathrm{~min}$ and subsequently washed with phosphate-buffered saline (PBS) 3 times for 3 min each. Following this, cells were blocked with 5\% normal goat serum (Boster Biological Technology, Ltd., Wuhan, China) for 30 min at room temperature. After thorough washing with PBS 3 times for 3 min each, the cells were incubated with anti-TROP2 monoclonal antibody (1:500; catalogue no. sc-376746; Santa Cruz Biotechnology, Inc., Dallas, TX, USA) overnight at $4^{\circ} \mathrm{C}$. Subsequent to 3 washes for 3 min each with PBS, the cells were stained with fluorescein isothiocyanate-conjugated anti-mouse immunoglobulin G (1:200; catalogue no. ZF-0312; Beijing Zhongshan Golden Bridge Biotechnology Co., Ltd., Beijing, China) for $1 \mathrm{~h}$ at room temperature. Fluorescence-labeled TROP2 was observed and photographed under a fluorescent microscope.

Transfection. In order to knockdown the expression of endogenous TROP2, lentivirus containing two small interfering (si)RNA sequences targeting TROP2 were designed and synthesized by Shanghai GenePharma Co., Ltd., (Shanghai, China). The siRNA sequences were as follows: TROP2-homo-1100, 5'-GCACGCTCATCTATTACCT-3'; and TROP2-homo-550, 5'-CCAAGTGTCTGCTGCTCAA-3'. The negative scramble control sequence was as follows: 5'-TTCTCCGAACGTGTC ACGT- $3^{\prime}$. The cells were seeded at $\sim 1.0 \times 10^{5}$ cells/well into 6-well plates and cultured at $37^{\circ} \mathrm{C}$ overnight under standard conditions. After $50 \%$ confluence was reached, the number of cells in a well was counted using a hemocytometer. TROP2 siRNA were transfected into cells in Opti-MEM (Invitrogen; Thermo Fisher Scientific, Inc., Waltham, MA, USA) at a multiplicity of infection $(\mathrm{MOI})=20[\mathrm{MOI}=$ transducing units per cell (TU) number/cell], according to the manufacturer's instructions. The culture medium was replaced after $24 \mathrm{~h}$ incubation. A total of $48 \mathrm{~h}$ after transfection, the cells were observed and photographed under a fluorescence microscope. After successful transfection, siRNA sequences were stably expressed. Untransfected cells were used as a blank control, while cells transfected with scrambled siRNA were considered as negative control.

Cell viability assay. Cell viability was examined using a cell counting kit-8 (CCK-8) assay based on the optical density (OD) value, according to the manufacturers protocol (Bestbio, Shanghai, China). Cells were seeded at $\sim 3.0 \times 10^{3}$ cells/well in a 96-well plate and cultured at $37^{\circ} \mathrm{C}$ in an incubator. After incubation for 24, 48, 72, 96 and $120 \mathrm{~h}, 10 \mu \mathrm{l}$ CCK-8 was added into each well, and subsequently incubated for another $3 \mathrm{~h}$ at $37^{\circ} \mathrm{C}$. The OD value was determined by the microplate reader at a wavelength of $450 \mathrm{~nm}$. The cell viability of each group was calculated by GraphPad Prism software, version 7.00 (GraphPad Software, Inc., La Jolla, CA, USA). The experiment was repeated three times.
Wound healing assay. Untransfected and transfected cells were seeded at $5.0 \times 10^{5}$ cells/well in 6-well plates and cultured routinely. After reaching $90 \%$ confluence, the cell monolayer was scratched with a sterile pipette tip. After washing 3 times with PBS for $5 \mathrm{~min}$ each to clear the floating cells, $1.5 \mathrm{ml}$ RPMI 1640 medium supplemented with 1\% FBS was added into each well. Photographs were taken by a microscope at 0,24 and $48 \mathrm{~h}$ after scratching. Results were indicated as the relative width of scratch-the distance migrated relative to the original scratched distance. The experiment was conducted three times.

Cell invasion assay. The invasive ability of cells was measured using the Corning ${ }^{\circledR}$ Matrigel ${ }^{\circledR}$ Basement Membrane Matrix (catalogue no. 356234; Corning Inc., Corning, NY, USA) and a 24-well transwell chamber (Corning Inc., Corning, New York, USA) according to the manufacturer's protocol. The number of cells that passed through an 8-mm polycarbonate membrane was calculated. The polycarbonate surface of each chamber was covered with $20 \mu 1$ Matrigel (1:4 dilution) to create an artificial basement membrane. Cells were cultured at $37^{\circ} \mathrm{C}$ in FBS-free RPMI 1640 medium for $24 \mathrm{~h}$. After serum starvation, the cells were seeded at $1 \times 10^{5}$ cells/well in the upper Transwell chamber, which contained $\sim 200 \mu 1$ serum-free RPMI 1640. The lower chamber was filled with $600 \mu \mathrm{l}$ of RPMI 1640 medium supplemented with 10\% FBS. After an incubation of $48 \mathrm{~h}$ at $37^{\circ} \mathrm{C}$, the chambers were fixed at room temperature with paraformaldehyde for $30 \mathrm{~min}$. Cells attached to the upper surface of the chambers were removed with a sterile cotton swab, and cells that adhered to the lower surface were stained with $0.1 \%$ crystal violet (Guangfu Institute of Superfine Chemical Industry, Tianjin, China) for $20 \mathrm{~min}$ at room temperature. The numbers of stained cells were counted using an inverted microscope (OLYMPUS IX 70-142; Olympus Corporation, Tokyo, Japan) in eight random fields. The experiment was repeated three times.

Western blot analysis. B-cell lymphoma 2 (Bcl-2) and Bcl-2-associated X protein (Bax) are two regulators of cell apoptosis, the former has a vital anti-apoptotic role and the latter has a pro-apoptotic role (15). Cellular proteins were extracted according to the protocol using a Total Protein Extraction kit (catalogue no. BB-3101; Bestbio) after a 48-h culture at $37^{\circ} \mathrm{C}$. Equivalent amounts $(20 \mu \mathrm{g})$ of protein samples were separated by $12 \%$ SDS-PAGE and transferred to polyvinylidene difluoride membranes. After blocking with 5\% $\mathrm{w} / \mathrm{v}$ non-fat dried milk for $1 \mathrm{~h}$ at room temperature, the blots were incubated with primary antibodies specific to TROP2 (catalogue no. sc-376746; Santa Cruz Biotechnology, Inc., Dallas, TX, USA), Bcl-2 (catalogue no. 4223; Cell Signaling Technology, Inc., Danvers MA, USA) and Bax (catalogue no. 5023, Cell Signaling Technology, Inc.; all 1:1,000) overnight at $4^{\circ} \mathrm{C}$. Subsequently, after 3 washes with PBS for $5 \mathrm{~min}$, the blots were incubated with horseradish peroxidase-conjugated goat anti-mouse $\operatorname{IgG}(\mathrm{H}+\mathrm{L})$ (catalogue no. ZB 2305; Beijing Zhongshan Golden Bridge Biotechnology Co., Ltd., Beijing, China; 1:4,000) for $1 \mathrm{~h}$ at room temperature. Bands were detected by enhanced chemiluminescence (EMD Millipore, Billerica, MA, USA) using ImageQuant LAS (General Electric Company, USA). The results were analyzed 


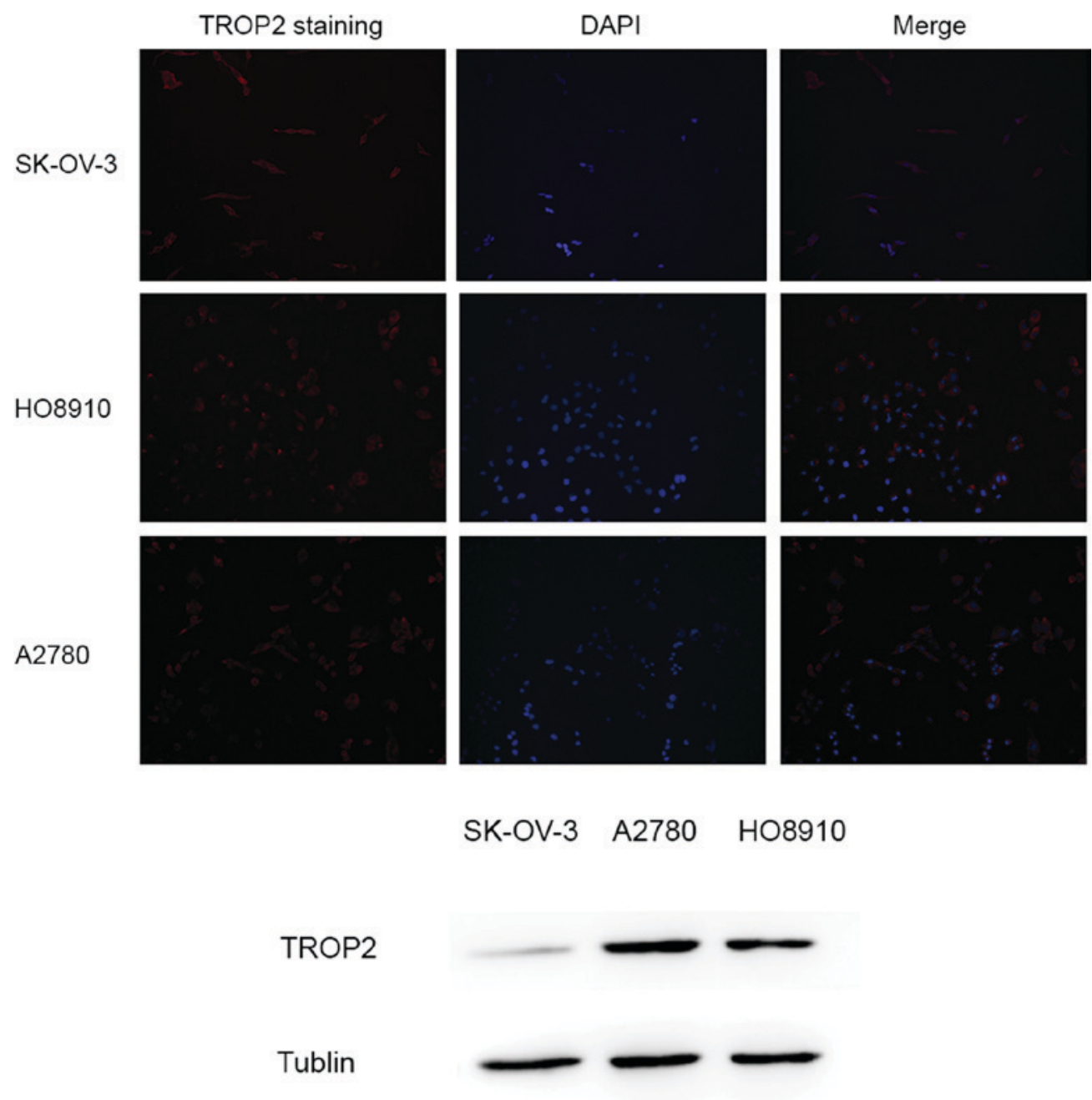

Figure 1. The expression of TROP2 in ovarian cancer cell lines. Immunofluorescence assay demonstrated a layer of red fluorescent staining on the membrane of SK-OV-3, HO8910 and A2780 cells, and the staining on A2780 cells was markedly brighter than the other two cell lines. Western blot analysis demonstrated the same result. TROP2, tumor-associated calcium signal transducer 2; DAPI, 4', 6-diamidino-2-phenylindole.

by Quantity One version 4.6.2 (Bio-Rad Laboratories, Inc., Hercules, CA, USA). GAPDH was used as an internal control.

Statistical analysis. Statistical analyses were performed using SPSS v. 17.0 software (SPSS, Inc., Chicago, IL, USA). Quantitative data were expressed as the mean \pm standard deviation. One-way analysis of variance and Student-Newman-Keuls tests were used. $\mathrm{P}<0.05$ was considered to indicate a statistically significant difference.

\section{Results}

Immunofluorescence staining of TROP2. Immunofluorescence analysis demonstrated a layer of red fluorescent staining on the cytomembranes of A2780, HO8910 and SK-OV-3 cells (Fig. 1). Western blotting results (Fig. 1) demonstrated that the A2780 cell line expressed markedly more TROP 2 protein than the other two cell lines.

Transfections. To investigate the specific functions of TROP2, the A2780 cell line was selected to conduct the following experiments. Two independent siRNA sequences were synthesized to downregulate the endogenous expression of TROP2. Cells transfected with scrambled siRNA were considered as the negative control group, and non-transfected cells were used as the blank control. Cells were observed and photographed by a fluorescence microscope $48 \mathrm{~h}$ after transfection. Transfected cells demonstrated a bright green stain (Fig. 2). The fluorescent staining indicated expression of green fluorescent protein, which implied successful transfection. A total of $48 \mathrm{~h}$ after transfection, the expression of TROP 2 protein in each group was measured. As demonstrated in Fig. 3A, the level of TROP2 protein in the transfected cells was significantly decreased, compared with the control groups $(\mathrm{P}<0.05)$. After transfection with sequence 1100 , the expression of TROP2 was significantly reduced compared with the sequence 550 group $(\mathrm{P}<0.05)$.

Cell viability assay. CCK- 8 assay was used to investigate the effect of TROP 2 on cell viability. Results demonstrated that the knockdown of TROP2 with the 550 and 1100 sequences induced a significant inhibition of the viability of A2780 cells compared with the control and negative control groups $(\mathrm{P}<0.05$; Fig. 3B and $\mathrm{C})$. These results indicated that TROP2 is required for the maintenance of cell viability. 


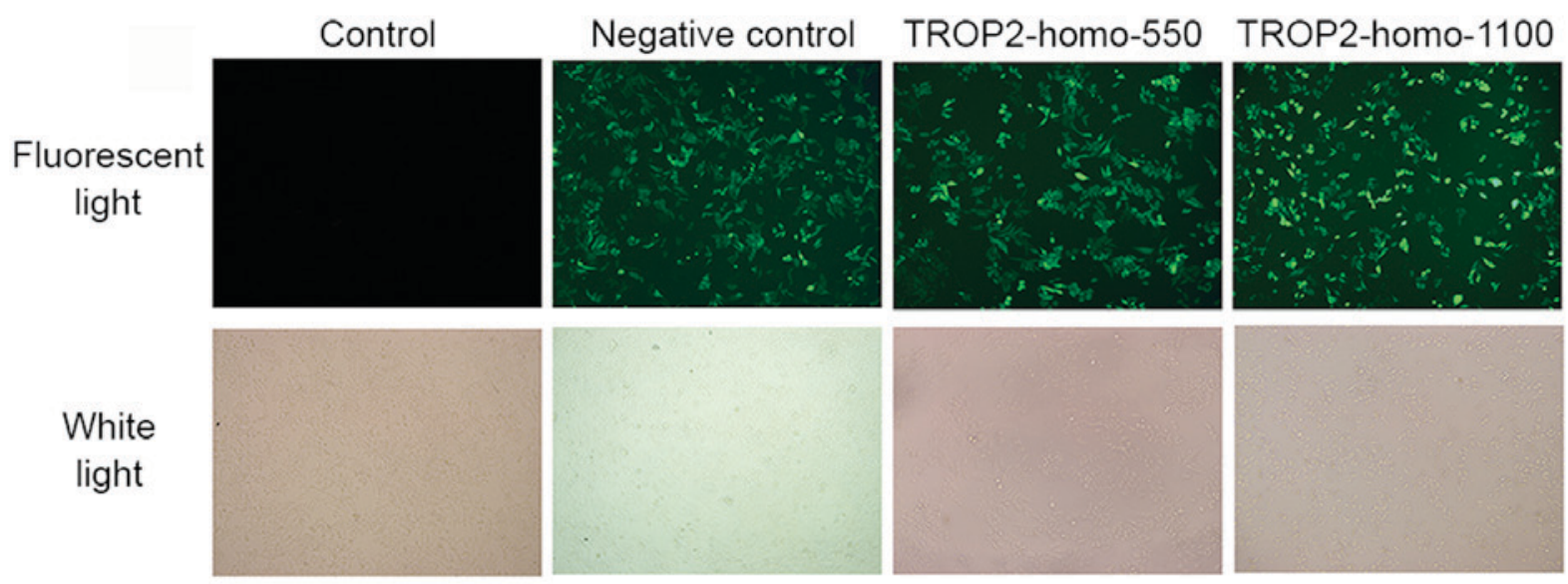

Figure 2. A2780 cells were observed and photographed by a fluorescence microscope $48 \mathrm{~h}$ after transfection with TROP2-homo-550 and TROP2-homo-1100 small interfering RNA sequences. The bright green stain for the expression of GFP protein implied successful transfection. TROP2, tumor-associated calcium signal transducer 2 .

A

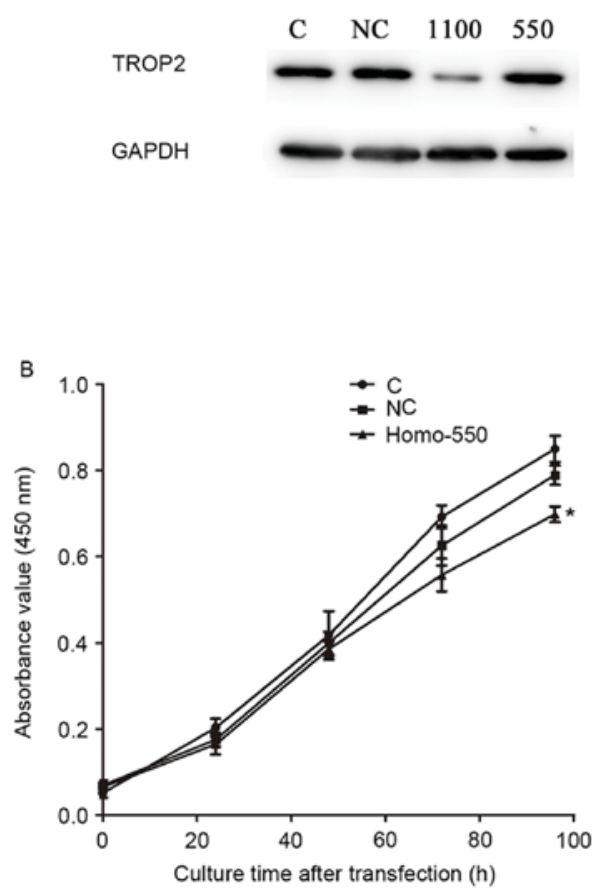

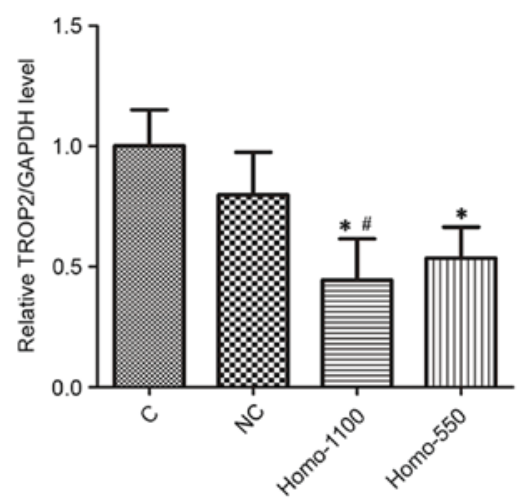

C

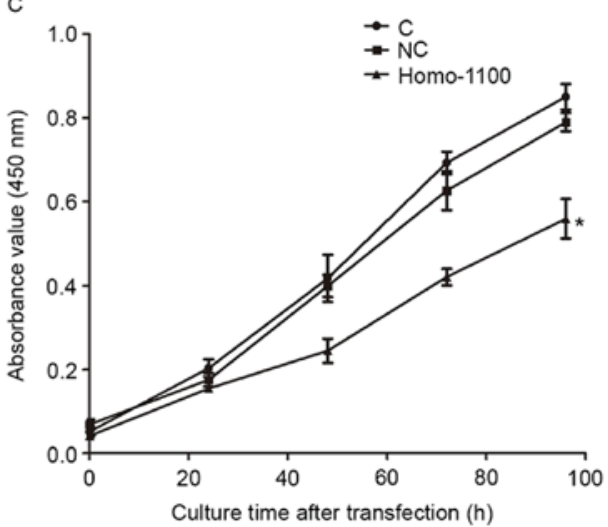

Figure 3. (A) Western blot analysis measured the effects of siRNA-mediated knockdown of TROP2. GAPDH was used as an internal control. Data are presented as the mean + standard deviation. Cells were transfected with (B) TROP2-homo-550 and (C) TROP2-homo-1100 siRNA and the viability was assessed using Cell counting kit-8 assay at five time points $(24,48,72,96$ and $120 \mathrm{~h}$ ). Transfected cells demonstrated a reduction in cell viability, and differences were significant when cells were cultured for $72 \mathrm{~h}$ (group 550) and $48 \mathrm{~h}$ (group 1100). Data are presented as the mean \pm standard deviation. ${ }^{*} \mathrm{P}<0.05 \mathrm{vs}$. the control groups, ${ }^{,} \mathrm{P}<0.05$ vs. group 550. TROP2, tumor-associated calcium signal transducer 2; siRNA, small interfering RNA; C, control; NC, negative control.

Wound healing assay. The monolayer wound healing assay was used to assess cell migration ability. As demonstrated in Fig. 4, 24 and $48 \mathrm{~h}$ after wounding, transfected cells indicated a significantly slower closing of the scratch, with a greater scratch width, compared with the control groups $(\mathrm{P}<0.05)$. These results indicated that TROP2 is required for cell migration.
Cell invasion assay. The serum-stimulated Matrigel invasion assay demonstrated that the percentages of A2780 cells that migrated through the polycarbonate membrane in the transfection groups were significantly lower than those in the control groups ( $\mathrm{P}<0.05$; Fig. 5). Therefore, downregulation of TROP2 decreased the invasive ability of A2780 cells at $48 \mathrm{~h}$ after transfection. 

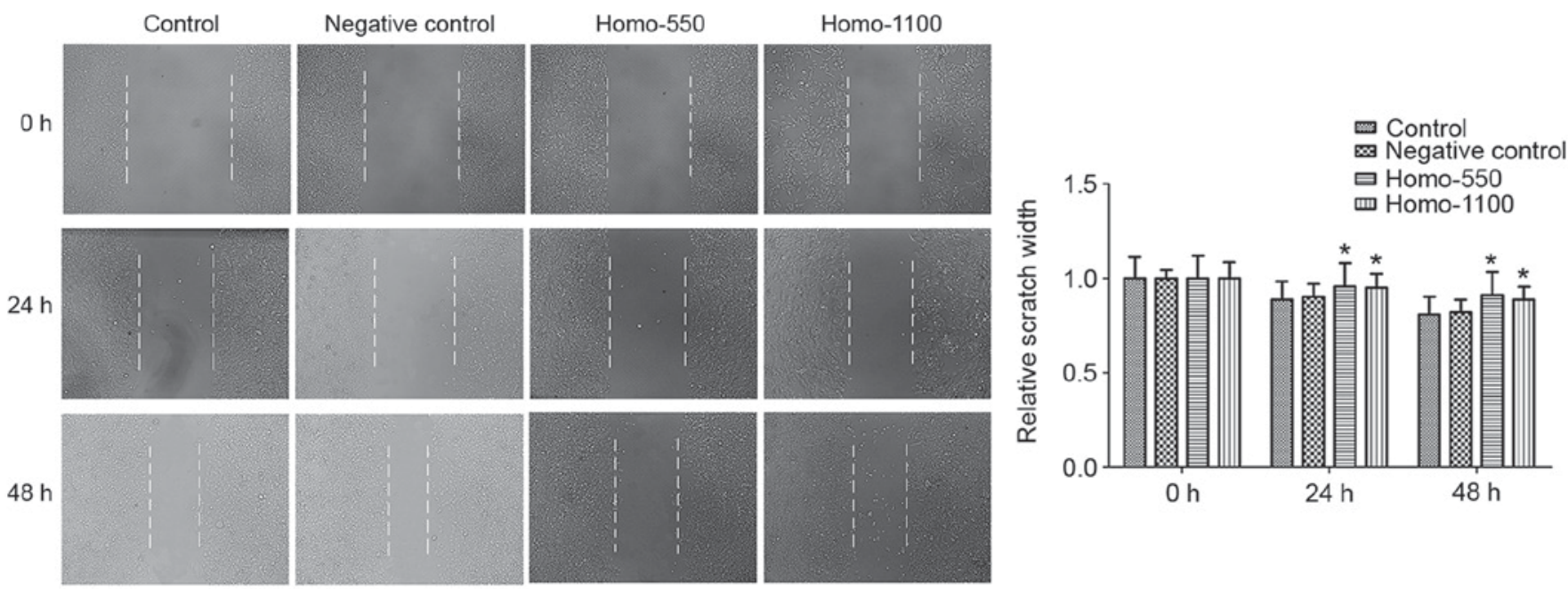

Figure 4. The effect of TROP2 expression on cell migration. Cells with TROP2-knockdown demonstrated slower wound recovery compared with the control groups at 24 and $48 \mathrm{~h}$ after wounding. Data are presented as the mean + standard deviation. ${ }^{*} \mathrm{P}<0.05 \mathrm{vs}$. the control groups at the corresponding time points. TROP2, tumor-associated calcium signal transducer 2 .
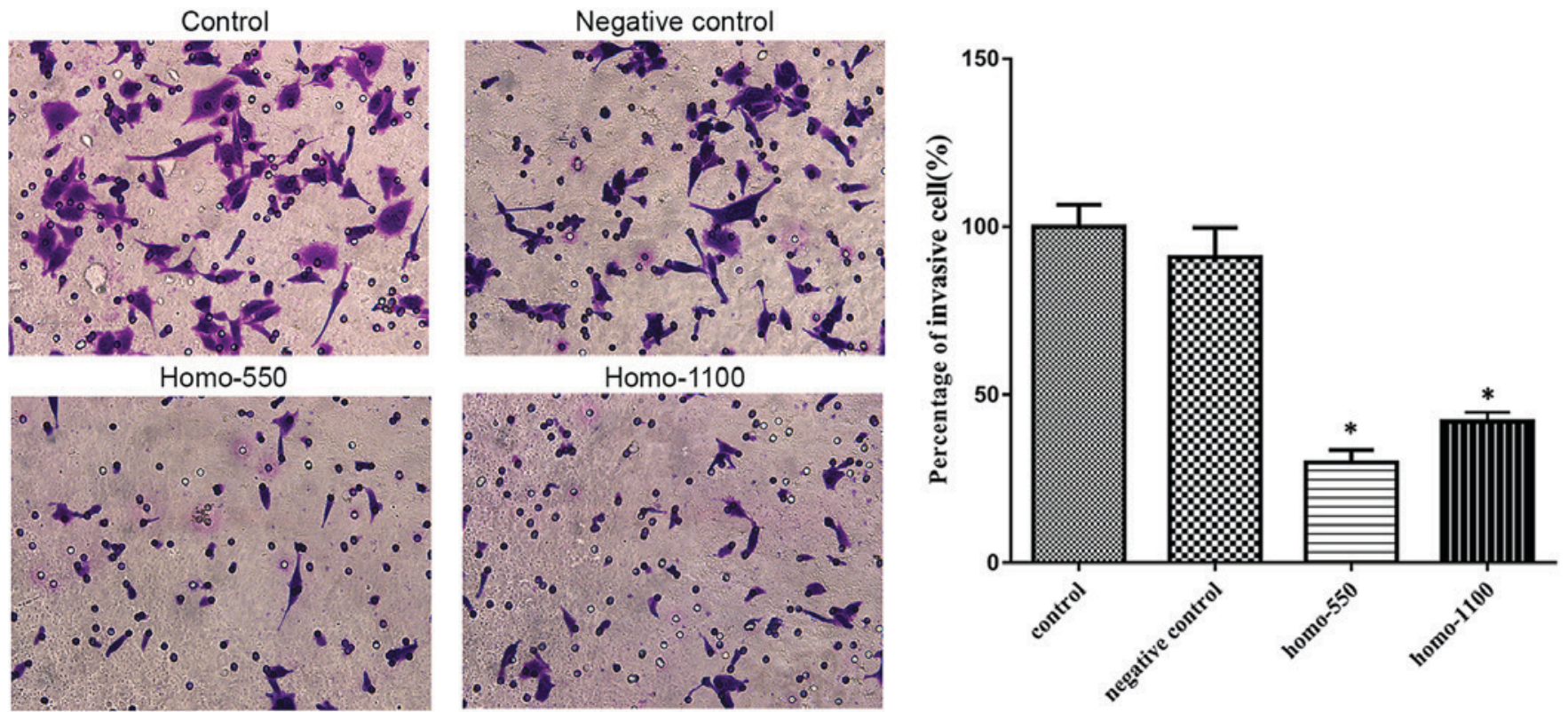

Figure 5. The influence of TROP2 expression on the invasive capability of A2780 cells. Downregulation of TROP2 inhibited cell invasion. Data are presented as the mean + standard deviation. ${ }^{*} \mathrm{P}<0.05$ vs. the control groups. TROP2, tumor-associated calcium signal transducer 2.

Western blot analysis. To explore the functional mechanism of TROP2, the expression of Bcl-2 and Bax proteins were investigated by western blotting (Fig. 6). The results demonstrated that, following the knockdown of TROP2, the expression of Bax was markedly enhanced compared with the negative control, whereas $\mathrm{Bcl}-2$ protein expression levels were markedly decreased compared with the negative control.

\section{Discussion}

Ovarian cancer was the fifth leading cause of cancer-related death among women in the United States in 2014 (1). More than half of the cases of ovarian cancer are highly invasive and insensitive to therapies, and are usually diagnosed at the
NC $\quad 550 \quad 1100$

Bax

Bcl-2

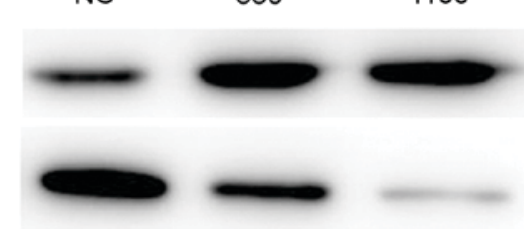

GAPDH

Figure 6. Western blot analysis of expression of Bcl-2 and Bax proteins GAPDH was used as an internal control. Following the knockdown of TROP2, the expression of Bax was enhanced, whereas Bcl-2 protein expression levels were decreased compared with the NC. Bcl-2, B-cell lymphoma 2; Bax, Bcl-2-associated X protein; $\mathrm{NC}$, negative control. 
advanced stages, resulting in a poor prognosis (16). As a type I transmembrane protein with several glycosylation sites, TROP2 is encoded by the single-exon gene, TACSTD2, which is expressed in various human carcinomas (4). However, the role of TROP2 is not well understood. Although TROP2 has previously been suggested to be involved in the adhesion between cancer cells, data has also indicated that it may have a role in cell signal transduction and the growth of cancer cells $(17,18)$.

In the present study, TROP2 expression and localization was investigated by immunofluorescence staining in A2780, HO8910 and SK-OV-3 cells. The results demonstrated that, compared with the other two cell lines, A2780 cells expressed a higher level of TROP2 protein. Therefore, the A2780 cell line was selected to conduct the subsequent experiments. siRNA was utilized to knockdown the endogenous expression of TROP2 to evaluate its effect. In vitro study demonstrated that the downregulation of TROP2 was able to suppress A2780 cell proliferation. By wound healing and Transwell invasion assays, it was indicated that the knockdown of the TROP2 gene was able to decrease the invasion and metastatic capabilities of A2780 cells. Anti-apoptosis was considered as a distinct characteristic of tumor development. Previous research has revealed that the balance between $\mathrm{Bcl}-2$ and Bax has a pivotal role in anti- and pro-apoptosis (15). The present study demonstrated that downregulated expression of TROP2 increased Bax expression and decreased $\mathrm{Bcl}-2$ expression in A2780 cells. The results of the present study therefore suggested that TROP 2 may be associated with the invasion, metastasis and resistance to apoptosis of A2780 cells. The effect of TROP2 on the inhibition of apoptosis may work via Bcl-2 family activation. However, there are limitations in the present study, a study by Domcke et al (19) has demonstrated that the A2780 cell line exhibited pronounced differences in molecular profiles vs. tumors, therefore the current research should be completed by subsequent experiments to verify the function and mechanism of TROP2 in an animal tumor-burdened model.

In conclusion, the present study demonstrated that silencing TROP2 expression in A2780 cancer cells suppresses proliferation, invasion and metastasis. Therefore, TROP2 has potential as a novel target for ovarian cancer treatment.

\section{References}

1. Siegel R, Ma J, Zou Z and Jemal A: Cancer statistics, 2014. CA Cancer J Clin 64: 9-29, 2014.

2. Cannistra SA: Cancer of the ovary. N Engl J Med 351: 2519-2529, 2004.

3. Auersperg N, Ota T and Mitchell GW: Early events in ovarian epithelial carcinogenesis: Progress and problems in experimental approaches. Int J Gynecol Cancer 12: 691-703, 2002.

4. Fong D, Spizzo G, Gostner JM, Gastl G, Moser P, Krammel C, Gerhard S, Rasse M and Laimer K: TROP2: A novel prognostic marker in squamous cell carcinoma of the oral cavity. Mod Pathol 21: 186-191, 2008.
5. Shvartsur A and Bonavida B: Trop2 and its overexpression in cancers: Regulation and clinical/therapeutic implications. Genes Cancer 6: 84-105, 2015

6. Bignotti E, Zanotti L, Calza S, Falchetti M,Lonardi S, Ravaggi A, Romani C, Todeschini P, Bandiera E, Tassi RA, et al: Trop-2 protein overexpression is an independent marker for predicting disease recurrence in endometrioid endometrial carcinoma. BMC Clin Pathol 12: 22, 2012.

7. Huang H, Groth J, Sossey-Alaoui K, Hawthorn L, Beall S and Geradts J: Aberrant expression of novel and previously described cell membrane markers in human breast cancer cell lines and tumors. Clin Cancer Res 11: 4357-4364, 2005.

8. Ohmachi T, Tanaka F, Mimori K, Inoue H, Yanaga K and Mori M: Clinical significance of TROP2 expression in colorectal cancer. Clin Cancer Res 12: 3057-3063, 2006.

9. Bignotti E, Ravaggi A, Romani C, Falchetti M, Lonardi S, Facchetti F, Pecorelli S, Varughese J, Cocco E, Bellone S, et al: Trop-2 overexpression in poorly differentiated endometrial endometrioid carcinoma: Implications for immunotherapy with hRS7, a humanized anti-trop-2 monoclonal antibody. Int J Gynecol Cancer 21: 1613-1621, 2011.

10. Varughese J, Cocco E, Bellone S, Ratner E, Silasi DA, Azodi M, Schwartz PE, Rutherford TJ, Buza N, Pecorelli S and Santin AD: Cervical carcinomas overexpress human trophoblast cell-surface marker (Trop-2) and are highly sensitive to immunotherapy with hRS7, a humanized monoclonal anti-Trop-2 antibody. Am J Obstet Gynecol 205: 567.e1-7, 2011.

11. Wang XD, Wang Q, Chen XL, Huang JF, Yin Y, Da P and Wu H: Trop2 inhibition suppresses the proliferation and invasion of laryngeal carcinoma cells via the extracellular signal-regulated kinase/mitogen-activated protein kinase pathway. Mol Med Rep 12: 865-870, 2015.

12. Bignotti E, Todeschini P, Calza S, Falchetti M, Ravanini M, Tassi RA, Ravaggi A, Bandiera E, Romani C, Zanotti L, et al: Trop-2 overexpression as an independent marker for poor overall survival in ovarian carcinoma patients. Eur J Cancer 46: 944-953, 2010.

13. Varughese J, Cocco E, Bellone S, Bellone M, Todeschini P, Carrara L, Schwartz PE, Rutherford TJ, Pecorelli S and Santin AD: High-grade, chemotherapy-resistant primary ovarian carcinoma cell lines overexpress human trophoblast cell-surface marker (Trop-2) and are highly sensitive to immunotherapy with hRS7, a humanized monoclonal anti-Trop-2 antibody. Gynecol Oncol 122: 171-177, 2011.

14. Bignotti E, Todeschini P, Calza S, Falchetti M, Ravanini M, Tassi RA, Ravaggi A, Bandiera E, Romani C, Zanotti L, et al: Trop-2 overexpression as an independent marker for poor overall survival in ovarian carcinoma patients. Eur J Cancer 46: 944-953, 2010.

15. Chen CR, Xia YH, Yao SY, Zhang Q, Wang Y and Ji ZN: Virosecurinine induces apoptosis by affecting Bcl-2 and Bax expression in human colon cancer SW480 cells. Pharmazie 67: 351-354, 2012.

16. Jordan S, Green A and Webb P: Benign epithelial ovarian tumours-cancer precursors or markers for ovarian cancer risk? Cancer Causes Control 17: 623-632, 2006.

17. Ripani E, Sacchetti A, Corda D and Alberti S: Human Trop-2 is a tumor-associated calcium signal transducer. Int J Cancer 76: 671-676, 1998.

18. Fornaro M, Dell'Arciprete R, Stella M, Bucci C, Nutini M, Capri MG and Alberti S: Cloning of the gene encoding Trop-2, a cell-surface glycoprotein expressed by human carcinomas. Int J Cancer 62: 610-618, 1995.

19. Domcke S, Sinha R, Levine DA, Sander C and Schultz N: Evaluating cell lines as tumour models by comparison of genomic profiles. Nat Commun 4: 2126, 2013. 